\title{
Aristote, De Cœlo, I 9 : l'identité des « êtres de là-bas »
}

Fabienne Baghdassarian

\section{(2) OpenEdition}

\section{Journals}

\section{Édition électronique}

URL : http://journals.openedition.org/philosant/1079

DOI : 10.4000/philosant.1079

ISSN : 2648-2789

\section{Éditeur}

Presses universitaires du Septentrion

\section{Édition imprimée}

Date de publication : 1 novembre 2011

Pagination : 175-203

ISBN : 978-2-7574-0356-3

ISSN : 1634-4561

\section{Référence électronique}

Fabienne Baghdassarian, «Aristote, De Cœlo, I 9 : l'identité des « êtres de là-bas » », Philosophie antique [En ligne], 11 | 2011, mis en ligne le 01 novembre 2018, consulté le 19 avril 2019. URL : http:// journals.openedition.org/philosant/1079; DOI : 10.4000/philosant.1079

Ce document a été généré automatiquement le 19 avril 2019

\section{(c) $(1)$}

La revue Philosophie antique est mise à disposition selon les termes de la Licence Creative Commons Attribution - Pas d'Utilisation Commerciale - Pas de Modification 4.0 International. 


\title{
Aristote, De Coelo, I 9 : l'identité des « êtres de là-bas »
}

\author{
Fabienne Baghdassarian
}

1 En De Colo $(D C)$, I, 9, c'est en montrant que notre monde utilise la totalité de la matière existante ${ }^{1}$ qu'Aristote choisit de défendre la thèse de l'unicité de l'univers ${ }^{2}$. On comprend, en effet, qu'il est impossible que plusieurs univers existent, s'il n'y a aucun substrat matériel qui puisse entrer dans la composition d'un autre monde que le nôtre. C'est pourquoi Aristote entreprend de démontrer qu'il ne se trouve rien de corporel au-delà de la limite dernière de notre ciel car, en vertu du nombre limité des corps élémentaires, en vertu de la théorie des lieux naturels, il faut admettre qu'aucun corps ne réside en dehors de l'univers ${ }^{3}$. Et si, poursuit-il, aucun corps ne s'y trouve, ne s'y trouvent pas non plus ces déterminations des corps que sont le lieu, le vide et le temps ${ }^{4}$. Assurément, on peut donc conclure qu'il n'existe pas, qu'il n'a jamais existé, ni n'existera jamais d'autres univers que le nôtre 5 .

2 Bien qu'il ait atteint son objectif, le chapitre I 9 ne se referme pourtant pas sur cette conclusion. Interviennent encore quelques remarques, par lesquelles Aristote procède à un éloge appuyé de la perfection de certains êtres. Il n'est pas rare, dans le $D C$, qu'Aristote conclue une argumentation cosmologique en recourant au témoignage de la tradition ou de la réflexion théologique. Il en va ainsi des remarques sur lesquelles s'achève, en $\mathrm{I} 3^{6}$, la démonstration de la supériorité de l'élément éthéré. C'est aussi ce à quoi l'on assiste, en I $9^{7}$, dans les dernières lignes de la preuve de l'unicité de l'univers. Aristote s'y réfère à la clairvoyance des Anciens ${ }^{8}$, puis aux enseignements des enquêtes théologiques ${ }^{9}$, afin de mieux souligner la perfection des "êtres de là-bas ", leur immutabilité, leur existence heureuse et autarcique, ainsi que l'éternité dont ils jouissent. Le procédé rhétorique et démonstratif du texte n'a donc rien d'inhabituel. Il n'est pas certain, cependant, qu'on en connaisse précisément l'objet. Sans nul doute, les êtres en question sont fort éloignés de nous. On en ignore, toutefois, l'identité exacte. Sur ce point, du reste, les interprètes ne sont jamais parvenus à s'unir au sein d'un consensus un peu ferme. Ils s'opposent, au contraire, depuis l'antiquité, entre partisans de deux hypothèses distinctes, certains 
penchant en faveur d'une référence aux astres fixes ${ }^{10}$, d'autres estimant que « les êtres de là-bas » désignent des réalités hypercosmiques ${ }^{11}$ :

C'est pourquoi les êtres de là-bas ( $\tau \dot{\alpha} \kappa \varepsilon \tilde{)})$ ne sont pas par nature dans un lieu, ni ne vieillissent par le temps, il n'y a pas non plus de changement pour aucun des êtres qui se rangent au-dessus de la translation la plus extérieure, mais inaltérables et impassibles, ils possèdent l'existence la meilleure et la plus autarcique, qu'ils poursuivent pendant toute leur durée de vie. (Et, en effet, les Anciens ont divinement parlé en prononçant ce nom. De fait, le terme qui enveloppe le temps de la vie de chacun, en dehors duquel on n'est pas conformément à la nature, on l'a appelé la durée de vie de chacun. Et, selon le même raisonnement, le terme du ciel entier, c'est-à-dire le terme qui enveloppe la totalité du temps et l'infini, c'est la

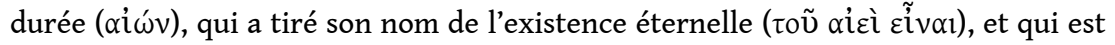
immortelle et divine.) C'est de là aussi, pour les autres êtres, que dépendent, plus régulièrement pour les uns, obscurément pour les autres, l'être et la vie. Et, en effet, comme dans les travaux philosophiques destinés au public et qui portent sur les réalités divines, on fait souvent valoir, grâce aux raisonnements, qu'il est nécessaire que le divin soit immuable dans sa totalité, lui qui est premier et suprême. Qu'il en soit ainsi témoigne en faveur de ce qu'on a dit. En effet, il n'y a rien de supérieur à lui qui le puisse mouvoir (car cela serait plus divin), il ne possède rien de vil, ni ne manque d'aucune des beautés qui lui sont propres. Il est aussi raisonnable qu'il soit

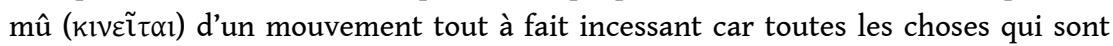
mues cessent leur mouvement chaque fois qu'elles arrivent dans leur lieu propre, mais, pour le corps mû en cercle, c'est le même lieu que celui d'où il part et celui où il achève son mouvement ${ }^{12}$.

3 À la lecture de la totalité du passage, on comprend le trouble qui est celui des interprètes : si les premières lignes, de 279a18 à 22 , suggèrent assez naturellement qu'il est ici question de réalités incorporelles extérieures à la sphère externe du ciel, la suite du texte, en revanche, ne paraît avoir de sens qu'à la condition qu'on estime que c'est l'heureuse perfection des étoiles fixes qu'Aristote décrit ici. N'est-ce pas en effet l'exemple du ciel qui, à partir de 279a25, motive la signification véritable de l'aíúv? Et n'est-ce pas assurément du ciel et de son mouvement circulaire qu'il est question, à partir de 27961 ? L'évidence textuelle de la transcendance des "êtres de là-bas» tend ainsi progressivement à s'effacer au profit de l'homogénéité du texte qui plaide en faveur d'une tout autre identité. Le scrupule de l'exégète renvoie alors dos à dos les indices textuels et les arguments structuraux, sans parvenir à les accorder.

4 Peut-être ce texte n'aurait-il pas tant piqué la curiosité des commentateurs, si l'on n'avait eu tendance à voir en lui le témoin précieux, bien qu'énigmatique, d'une certaine phase de la cosmologie et de la théologie d'Aristote, le tout sur fond d'une relecture chronologique du corpus. C'est un fait, en l'occurrence, qu'on s'étonne de ne pas voir apparaitre plus souvent la doctrine du Premier Moteur Immobile, dans le $D C$, c'est-à-dire dans un traité de cosmologie ${ }^{13}$ où les recherches d'Aristote le conduisent, de surcroît, à considérer de plus près les principes des déplacements stellaires ${ }^{14}$. Il est vrai que, en $D C$ II, $6^{15}$ et II, $12^{16}$, il énonce très clairement la subordination du mouvement astral à l'influence d'un moteur incorporel et parfait. Ces deux textes, toutefois, forment comme une exception à la règle qui veut que l'étude du ciel, dans le $D C$, ne mobilise jamais l'influence du Premier Moteur, de sorte que ces deux passages sont parfois suspectés d'être des ajouts $^{17}$ au corps principal de l'enquête. On saisit alors mieux l'importance que peut revêtir, dans ce contexte, l'intelligence de la fin du chapitre 9 du premier livre. Comprendre qui sont "les êtres de là-bas", trancher entre l'hypothèse de leur transcendance et celle de leur identité astrale, ce serait un peu parvenir à déterminer si la 
cosmologie du $D C$ recouvre parfaitement l'ensemble du réel ou bien si elle laisse ouverte la possibilité qu'il existe certaines réalités hypercosmiques. Il s'est souvent agi alors, pour les différents commentateurs du traité ${ }^{18}$, de valider ou d'invalider, au moyen de ce texte, l'hypothèse qu'Aristote ait pu, dans le $D C$, adhérer à ce qu'on peut appeler une théologie purement cosmique, dans laquelle les astres sont les réalités dernières et les divinités les plus hautes.

5 À cet égard, il est remarquable que, à quelques exceptions près ${ }^{19}$, la tradition interprétative n'ait abordé cette question qu'au moyen d'une logique binaire : soit, en effet, le texte confirme qu'il n'y a rien au-delà des astres fixes et l'on en conclut alors très légitimement que le Stagirite ne croyait pas nécessaire de s'en remettre à la doctrine du Premier Moteur Immobile; soit le texte atteste l'existence de certaines réalités transcendantes et c'est alors au Premier Moteur qu'elles sont assez immédiatement rattachées. En somme, tout se passe comme si Aristote n'avait pas d'autre moyen, pour s'en référer à l'existence d'êtres métaphysiques, que d'en passer par la théorie du Premier Moteur. Si cette alternative est la bonne, s'il est légitime de conclure que les textes qui admettent l'existence d'une réalité transcendante admettent ipso facto celle du Premier Moteur, c'est alors que ce concept relève principalement d'un ordre théologique du discours : ce serait donc à sa perfection et à sa transcendance, plus qu'à sa fonction motrice, qu'il faudrait accorder le plus de valeur. Dans cette perspective, la doctrine du Premier Moteur serait bien plus que l'affirmation de l'existence d'un principe immobile du mouvement céleste; elle serait aussi et surtout ce par quoi Aristote poserait l'existence d'un être divin transcendant. En revanche, s'il est envisageable de basculer vers une logique ternaire, s'il est envisageable qu'Aristote ait pu souscrire à l'existence d'une ou plusieurs réalités transcendantes qui ne fussent pas pour autant des moteurs immobiles, il faut alors accorder plus de poids à la fonction du Premier Moteur et, ainsi, prendre en compte son inscription au sein d'un discours archéologique, puisque dès lors la doctrine qui l'énonce ne servirait pas tant à poser la thèse de la transcendance divine qu'à mieux définir la fonction cosmique de cette transcendance.

6 Relativement à cette question, il peut sembler souhaitable d'entreprendre à nouveaux frais l'analyse de ce passage du $D C \mathrm{I}, 9$, car il n'est pas impossible qu'il permette d'apprécier le domaine de pertinence de la doctrine du Premier Moteur, en jetant quelque lumière, si faible soit-elle, sur ses motivations conceptuelles. Ceci, toutefois, n'est envisageable qu'à la condition qu'on parvienne à déterminer, avec certitude, qui sont les êtres de là-bas, ce qui - on le mesure à la complexité du passage - implique qu'on réussisse à faire converger à la fois le contexte de l'extrait, sa formulation et sa structure.

\section{La démonstration en $D C \mathrm{I}, 9$ et la recherche de l'extériorité}

7 DC I, 9, avons-nous dit, est tout entier consacré à la démonstration de l'unicité de l'univers. Cette question n'est pas nouvelle; son traitement remonte au début du chapitre $8^{20}$. À l'inverse du chapitre 8, cependant, Aristote n'entreprend pas de prouver, au moyen d'un raisonnement par l'absurde, que l'univers est unique, en montrant les conséquences inadmissibles qu'engendrerait l'existence de mondes multiples ${ }^{21}$; il souhaite plutôt ruiner à sa source l'hypothèse même de la multiplicité des mondes. À cet effet, il soutient que le monde fait exception à la règle selon laquelle tous les êtres sensibles peuvent être numériquement multiples, quoique formellement identiques ${ }^{22}$. 
Sans récuser la validité générale de ce principe, il entend prouver qu'il ne s'applique pas au cas particulier de l'univers, étant donné que celui-ci utilise déjà la totalité de la matière existante ${ }^{23}$. Ainsi, la multiplicité des univers n'est pas exclue de droit, mais elle est impossible de fait ${ }^{24}$. À ce stade, cependant, Aristote n'a encore rien prouvé : il a simplement indiqué la piste qu'il souhaitait suivre dans le but de démontrer l'unicité du ciel. En l'occurrence, suivre cette piste requiert de prouver que rien n'existe en dehors du ciel $^{25}$, du moins, rien de corporel, rien qui puisse constituer une matière disponible pour la constitution d'un autre monde. À partir de $278625^{26}$, Aristote en fait la démonstration :

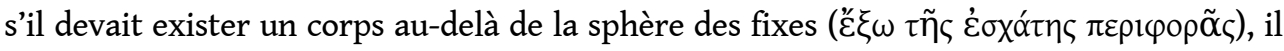
s'agirait soit d'un corps simple, soit d'un corps composé. Dans la première hypothèse, ce corps devrait s'y trouver soit par nature, soit contrairement à la nature. Qu'il y soit par nature, cela est impossible, puisque le lieu propre de chacun des cinq éléments est à l'intérieur du monde. Il n'est pas non plus possible qu'il y soit contre nature, puisque ce qui est contre nature pour un corps est naturel pour un autre corps. Or, il n'existe pas de sixième élément ${ }^{27}$. Aucun corps simple ne peut donc se trouver à l'extérieur du monde et, en conséquence, aucun corps composé non plus, puisque si un corps composé s'y trouve, un corps simple au moins doit s'y trouver aussi. Il ne peut donc ni ne pourra y avoir plus d'un monde. Cette argumentation rejoint en partie celle du fragment 19a (Ross) du De Philosophia (DP), qui souhaitait démontrer, pour une autre raison ${ }^{28}$, que rien de corporel n'existait à l'extérieur du dernier orbe. Or, dans le fragment 19a, cette démonstration n'interdisait pas qu'Aristote envisageât qu'une réalité incorporelle pût exister à l'extérieur du ciel :

Si donc il existe quelque chose à l'extérieur du monde, ce serait un vide total ou bien une nature impassible qui ne pourrait ni pâtir ni agirir ${ }^{29}$.

8 Aussi peut-on s'attendre à ce qu'Aristote fasse allusion, en $D C$ I, 9 - comme ce fut le cas dans le $D P$ - au fait que son argumentation n'interdit pas l'existence hypercosmique d'une réalité incorporelle. Dans ces deux textes, en tout cas, la réfutation du Stagirite porte exclusivement sur la possibilité que quelque chose de corporel existe à l'extérieur du monde. En témoigne le corollaire qu'il énonce, en 279a11-18, par lequel il exclut que les propriétés physiques des corps existent en dehors de l'univers :

En même temps, il est clair qu'il n'y a ni lieu, ni vide, ni temps en dehors du ciel ${ }^{30}$.

Le lieu, en effet, est la place du corps naturel, tandis que le vide - dont Aristote admet ici l'existence - en signale l'absence. Quant au temps, il est nécessairement lié au mouvement et, par suite, à l'existence d'un corps susceptible de se mouvoir. Si donc il n'y a aucun corps à l'extérieur du ciel, il n'y a ni place, ni temps, dans lesquels il pourrait résider. Ce qui est en dehors du ciel ne relève donc plus de l'ordre physique. Remarquons toutefois que ceci n'élimine pas totalement la possibilité que quelque chose de métaphysique puisse y exister, pas plus que n'empêche de soutenir, en Physique, VIII, l'existence d'une réalité transcendante, l'argumentation qu'Aristote mène en Physique, IV, 5, par laquelle il prouve que le ciel n'est nulle part, puisqu'il n'y a rien en dehors de lui. Cette remarque a son importance car c'est immédiatement après avoir souligné qu'il n'y avait ni lieu, ni vide, ni temps en dehors du ciel qu'Aristote en vient à énoncer l'existence de réalités, au sujet desquelles on s'interroge pour savoir si elles sont transcendantes :

Il est donc manifeste qu'il n'y a ni lieu, ni vide, ni temps hors du ciel. C'est pourquoi les êtres de là-bas ne sont pas par nature dans un lieu, ni ne vieillissent par le temps, il n'y a pas non plus de changement pour aucun des êtres qui se rangent audessus de la translation la plus extérieure ${ }^{31} \ldots$ 

est une conséquence ( $\Delta$ ó́ $\varepsilon \rho)$ de la remarque selon laquelle il n'y a ni lieu, ni temps, ni vide en dehors du ciel. C'est pourquoi Jaeger ${ }^{32}$, Guthrie ${ }^{33}$ et Allan $^{34}$ ne ponctuent pas comme le fait Moraux (dont nous reproduisons ici le texte) : contrairement à Moraux qui sépare ces deux remarques par un point, pour mieux suggérer qu'elles sont déconnectées l'une de l'autre, ils préfèrent placer un point en haut, accentuant ainsi la continuité de l'argumentation. Et c'est précisément cette continuité que Simplicius ${ }^{35}$ fait valoir en faveur de l'existence de réalités transcendantes : il lui apparaît qu'Aristote est ici en train d'énoncer les corollaires de l'inexistence du lieu, du vide et du temps en dehors de l'univers, pour déterminer le statut de ce qui n'est ni dans le temps, ni dans le lieu ${ }^{36}$. Il n'y a pas nécessairement de contradiction à décrire des êtres transcendants à la manière d'Aristote ici, c'est-à-dire au moyen de concepts corporels (le lieu et le temps), car l'analyse du contexte immédiat de cet extrait a pu convaincre que l'attention du Stagirite se portait uniquement sur la possibilité de l'existence d'un corps hypercosmique. Cela n'a donc rien d'incompréhensible qu'il examine ce qu'il en est des réalités hypercosmiques incorporelles au moyen des seuls concepts dont il dispose ${ }^{37}$. On pourrait toutefois estimer que cette continuité argumentative ne prouve rien, attendu que le ciel, au sens strict, n'est ni dans un lieu, ni dans le temps et qu'il peut, en outre, être dit immuable. Qu'il ne soit pas dans le temps, ni ne vieillisse en lui, la relation du mouvement astral au temps doit nous en convaincre. L'existence des astres fixes ne peut être mesurée par le temps, puisque c'est leur mouvement qui en est la mesure ${ }^{38}$. Que le ciel ne soit pas dans un lieu, c'est ce qu'Aristote explique en Phys. IV, 5, 21267 sqq., lorsqu'il montre que le ciel, en général, et la sphère des fixes, en particulier, ne sont pas dans un lieu, dans la mesure où il n'y a rien d'extérieur à eux qui les puisse envelopper.

En effet, ce qui est quelque part est lui-même quelque chose et, en outre, il faut qu'il y ait quelque chose d'autre à côté de lui, dans lequel il est, qui l'enveloppe. Or, à côté de l'univers et du tout, il n'y a rien en dehors de l'univers et, pour cette raison, toutes les choses sont dans le ciel. Le ciel, en effet, est vraisemblablement l'univers. Or, le lieu n'est pas le ciel, mais c'est l'extrémité du ciel qui touche le corps mobile. Et pour cette raison, la terre est dans l'eau, celle-ci est dans l'air, celui-ci dans l'éther, l'éther est dans le ciel, tandis que le ciel n'est dans rien d'autre ${ }^{39}$.

Manifestement, ce passage de Phys. IV soutient que le ciel n'est pas dans un lieu. Remarquons cependant que cela concerne uniquement la sphère des fixes, prise comme un tout. Or, même dans l'hypothèse où " les êtres de là-bas ", en $D C \mathrm{I}, 9$, désigneraient des réalités célestes, il faudrait encore admettre que ce n'est pas de la sphère des fixes qu'il s'agit, mais de la myriade d'étoiles qui sont fixées sur elle et qui en constituent comme des parties. Or, si la sphère externe n'est assurément nulle part, on peut estimer, à l'inverse, que chaque étoile fixe est dans un lieu, attendu qu'elle est entourée, non seulement par la matière éthérée qui constitue l'orbite sur laquelle elle est posée, mais encore, par chacune des autres étoiles fixes qui résident à ses côtés. Par suite, il n'est pas certain que l'absence de lieu, qui sert à caractériser « les êtres de là-bas ", puisse convenir aux réalités célestes aussi bien qu'aux réalités supracélestes. Il peut sembler, au contraire, qu'Aristote énonce là une propriété qui ne se peut comprendre qu'au sujet d'êtres transcendants à la sphère des fixes. Peut-être en va-t-il de même de la parfaite

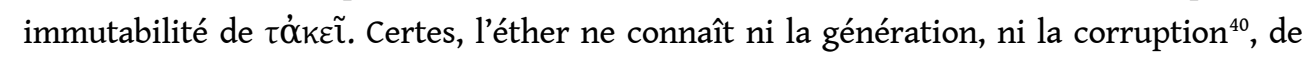

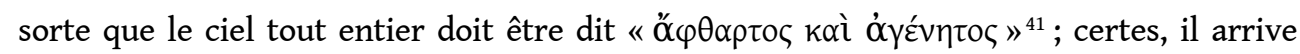
aussi qu'Aristote décrive le ciel comme totalement immuable ${ }^{42}$, compte tenu du fait que son mouvement n'engendre aucun changement d'état; il n'en demeure pas moins que le

Philosophie antique, 11 | 2011 
ciel connaît une forme de changement : le mouvement local en cercle. Or, notre texte ne se contente pas d'énoncer la simple immutabilité des "êtres de là-bas »: il insiste, au

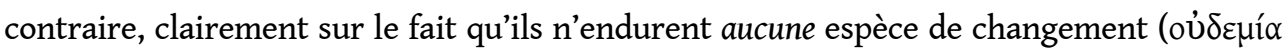
$\left.\mu \varepsilon \tau \alpha \beta \beta_{0} \eta_{\eta}\right)$. Cette insistance est peut-être plus qu'un effet de style ${ }^{43}$. Elle peut introduire une précision, par laquelle Aristote sous-entendrait que les êtres dont il parle ne sont pas immuables à la façon du ciel, mais plus fondamentalement encore, en n'étant pas même soumis au déplacement circulaire. Par suite, ce ne serait pas seulement l'enchaînement argumentatif de notre texte et de ce contexte immédiat qui plaiderait en faveur de

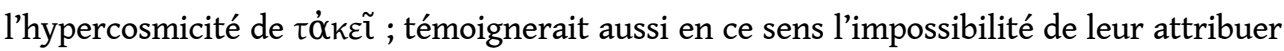
ni lieu, ni temps, ni aucun changement. À tous égards, il semble donc que le texte suit une ligne argumentative à la fois plus naturelle et plus directe, si l'on estime qu'Aristote passe logiquement de la considération selon laquelle il n'y a ni lieu ni temps en dehors de l'univers, à l'analyse de ce qui existe en dehors de l'univers, sans que le lieu, le temps, ni aucune sorte de changement ne le caractérisent.

\section{La description des êtres de là-bas}

12 S'il apparaît que la question de $\tau \dot{\alpha} \kappa \varepsilon \tilde{\imath}$ est directement motivée par la remarque selon laquelle il n'y a ni lieu, ni vide, ni mouvement, ni temps en dehors de l'univers, Aristote ne se contente toutefois pas de décrire ces êtres, en leur refusant simplement ces quelques propriétés corporelles. En quelques lignes, il précise, non seulement où se trouvent les réalités qui n'existent ni dans le lieu ni dans le temps, mais encore quelle est la perfection de leur existence. De ces deux perspectives, il ressort assez clairement que les êtres en question ne sont pas corporels, ni n'appartiennent au monde, mais qu'ils transcendent la sphère externe du ciel.

\section{La description topographique}

13 Lorsque Aristote souhaite préciser où sont ces êtres, au sujet desquels les déterminations locales, temporelles et cinétiques sont inappropriées, c'est à l'extériorité même du ciel

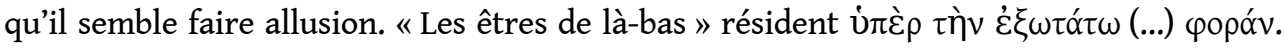
Sans doute faut-il commencer par remarquer que cette description accentue le paradoxe qu'il y a à décrire, par des motifs locaux, ce qui n'a pas de lieu. Aristote ne se contente plus ici de dire, par la négation, ce qu'il en est de certaines réalités hors normes; il semble attribuer positivement un lieu à ce qui ne peut en avoir. Cela, toutefois, ne

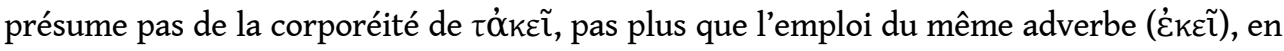
Phys. VIII, 10, 267b9, pour attribuer une place au Premier Moteur, ne doit faire douter de son immatérialité. Dire d'un être qu'il est là-bas, ce n'est pas tant déterminer son lieu que souligner sa transcendance. Notons, en outre, que ce ne semble pas véritablement être un lieu que désigne ici Aristote : « les êtres de là-bas » ne sont pas tant en un lieu que là où il n'y a plus de lieu; il ne résident pas quelque part, mais au-delà de la limite dernière, au moyen de laquelle on définit le fait d'être quelque part.

14 À première lecture, la lettre même du texte ne semble donc laisser planer aucun doute : « les êtres de là-bas » seraient au-delà de la sphère dernière. Deux éléments d'analyse semblent toutefois pouvoir atténuer cette première impression. Le premier tient au sens qu'il convient d'attribuer à la préposition u̇đźp : bien que la quasi-totalité des traducteurs choisisse de suivre le sens le plus évident de ن̇đép et de le traduire par «au-dessus $»^{44}$, 
Moraux, qui considère que ce texte se réfère aux astres, préfère rendre U̇đép par « sur » ${ }^{45}$. Les choses de là-bas désigneraient donc, selon lui, les astres fixes qui sont posés sur l'orbite la plus extérieure du ciel. Soulignons, toutefois, que Moraux est le seul à rendre le texte ainsi: même Alexandre d'Aphrodise ${ }^{46}$, qui penche pourtant en faveur de la référence astrale, comprend le texte comme caractérisant quelque chose qui est au-dessus d'un mouvement. Il est vrai que, dans les lignes qui précèdent, Aristote ne se réfère jamais à quoi que ce soit d'extérieur au monde au moyen de la préposition u̇đźp. Il préfère utiliser le terme $\varepsilon \xi \xi \omega^{47}$ qui n'a rien d'ambigu. C'est pourquoi on peut être tenté de déduire de ce partage terminologique que ce qui est úđźp n'est pas au-dessus de la dernière sphère. Toutefois, rien ne permet d'estimer que ce partage soit une loi. La préposition ữép, lorsqu'elle est employée avec l'accusatif, désigne ce qui est au-dessus de quelque chose et non pas ce qui est sur lui. En outre, chacune des occurrences de cette préposition dans le $D C$, qu'elle s'accompagne de l'accusatif ou même du génitif, sert constamment à désigner ce qui est au-dessus d'un point de référence. Enfin, c'est toujours la préposition $\dot{\varepsilon} v$, et non pas ữép, qui sert à désigner les corps qui sont fixés sur l'orbite ${ }^{48}$. À ces arguments, nous pourrions ajouter que la locution $\tau \dot{\alpha} \kappa \varepsilon \tilde{l}$ ( $\tau \grave{\alpha} \dot{\varepsilon} \kappa \varepsilon \tilde{l})$ semble par elle-même désigner des êtres extérieurs à notre monde. Il existe seulement deux occurrences de cette locution, dans l'ensemble du corpus : celle qui nous intéresse à présent et celle que l'on trouve en $D C \mathrm{I}, 8,267 \mathrm{~b} 3$, où elle désigne très clairement les corps qui, dans l'hypothèse où plusieurs mondes existeraient, seraient extérieurs à notre univers. Par suite, on peut raisonnablement incliner à penser que l'occurrence de I 8 justifie que le même terme, un chapitre plus loin, puisse à nouveau servir à désigner des êtres hypercosmiques. Du reste, il pourrait paraître étrange qu'Aristote, pour indiquer qu'il souhaite traiter des étoiles fixes, ait recours à une locution si vague et si peu habituelle.

Cette analyse, cependant, ne prouve pas définitivement que les êtres qui sont au-dessus de la translation la plus extérieure soient des êtres hypercosmiques, car il est un autre élément de la description topographique des "êtres de là-bas " qui peut prêter à discussion. De fait, Aristote n'emploie pas ici l'expression qui, en $D C \mathrm{I}$, 9, et même au-delà,

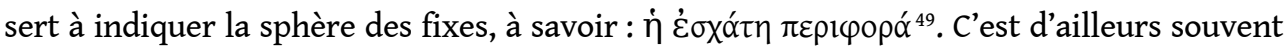
l'adjectif Ě $\sigma \alpha_{\alpha} \alpha{ }^{50}$ qui sert à décrire la sphère des fixes, quand Aristote ne choisit pas tout simplement de la désigner par la mention de sa primauté ${ }^{51}$. Deux éléments font donc exception à la règle : non seulement Aristote parle plus généralement d'une translation, plutôt que d'une révolution, mais en plus il préfère se référer à quelque chose d'extérieur, plutôt qu'à l'extrémité de l'univers. C'est pourquoi Alexandre ${ }^{52}$ fait l'hypothèse que le texte ne désigne pas ce qui est au-dessus du mouvement circulaire, mais le mouvement circulaire lui-même qui est au-dessus du mouvement rectiligne. À dire vrai, ces différences dans l'expression sont sans doute minimes : le remplacement de $\pi \varepsilon \rho 1 \varphi \circ \rho \alpha ́$ par

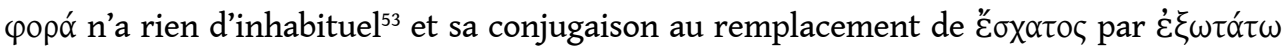
ne permet pas de douter qu'Aristote souhaite désigner la limite dernière du ciel. Peut-être même cette deuxième substitution se justifie-t-elle en raison de la première : attendu qu'il mentionne, sans autre précision, la translation en général, Aristote prendrait peutêtre soin de montrer qu'il entend désigner une translation circulaire, en insistant plus que de coutume sur l'extériorité maximale de cette $\varphi$ oó. Nul doute en tout cas que, si Aristote souhaitait désigner les astres fixes, il s'y serait pris autrement qu'en mobilisant une expression tout à fait inhabituelle. L'originalité de la formule peut donc convaincre, en un sens, qu'elle sert à désigner les êtres situés au-dessus de la limite du ciel et non pas ceux qui sont fixés sur elle. 


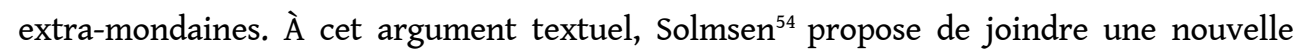

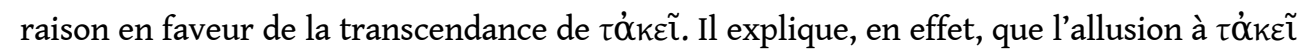
est sans doute un souvenir de deux passages des dialogues platoniciens. Dans le Phèdre, en $247 c$, on peut lire, en effet, que l'espace dans lequel résident les essences est au-delà du

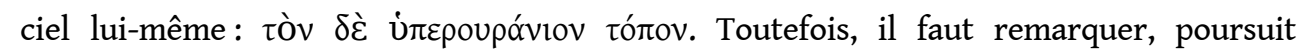
Solmsen, que « les êtres de là-bas » ne sont pas, chez Aristote, dans un lieu, précisément parce qu'il n'y a pas de lieu en dehors du ciel. Le Phèdre, en revanche, parle d'un lieu hypercéleste ( contre-argument, dans la mesure où le Timée explique, en $52 b$, que l'être véritable n'est dans aucun lieu. Par suite, qu'Aristote mentionne l'existence de réalités situées au-delà de la sphère externe du ciel, immédiatement après avoir expliqué qu'il n'existait aucun lieu en dehors du ciel, serait un prolongement (somme toute, très orthodoxe) du Phèdre et de la correction qu'y apporte le Timée. Dans l'optique de Solmsen, la transcendance de $\tau \dot{\alpha} \kappa \varepsilon \tilde{l}$ ne ferait plus aucun doute. Disons, en tout cas, que cette démonstration renforce sensiblement cette interprétation en éclairant quelques-uns des fondements philosophiques de ce passage et en montrant que cette référence aristotélicienne, sous le

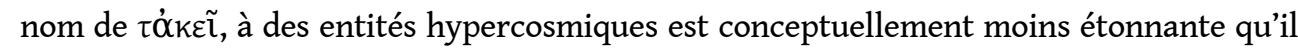
n'y paraît, puisqu'elle constituerait une référence à un aspect précis de la doctrine platonicienne.

\section{L'éloge de leur perfection}

Parce qu'elles n'existent pas dans un lieu, ni ne vieillissent dans le temps, parce qu'elles sont exemptes de changement, les réalités de là-bas jouissent d'une perfection telle qu'Aristote la souligne abondamment. Aussi sont-elles dites inaltérables ( $\dot{\alpha} v \alpha \lambda \lambda o i ́ \omega \tau \alpha)$, impassibles ( $\dot{\alpha} \pi \alpha \theta \tilde{\eta})$, et menant sans interruption, pour l'éternité entière, une vie parfaite

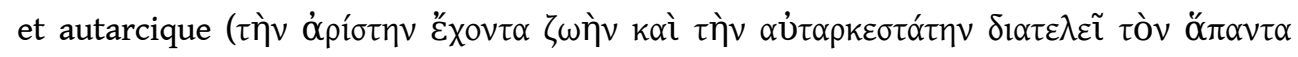
$\alpha i \tilde{\omega} v \alpha)$. Aucune de ces déterminations n'exclut définitivement l'hypothèse de la référence astrale. En effet, pas plus qu'ils ne connaissent la génération ou la corruption, l'éther et les corps qui en sont composés ne connaissent l'altération ${ }^{55}$. Quant à l'impassibilité du ciel, Aristote l'énonce régulièrement ${ }^{56}$, comme c'est le cas en $D C$ II, 1 , où il attribue au ciel les mêmes propriétés que celles qui nous occupent à présent. Il semble même qu'on puisse lire, dans ce chapitre, la perfection et l'autarcie de la vie des « êtres de là-bas ». Attendu que le mouvement des astres est tout à la fois éternel et naturel, Aristote estime qu'il ne leur procure aucune fatigue ${ }^{57}$ et qu'ils ont donc part à la disposition la meilleure ${ }^{58}$. Car il n'y a pas à douter que les astres possèdent la vie et l'activité ${ }^{59}$, une activité faite de félicité ${ }^{60}$ et de loisir intellectuel ${ }^{61}$. Ce sont, en somme, à peu près les mêmes termes que ceux qui, en $D C \mathrm{I}$, 9, servent à qualifier les « êtres de là-bas ».

Cependant, chacune de ces perfections fait plus naturellement songer à des réalités transcendantes. De fait, le livre $\Lambda$ abonde en descriptions fort similaires: en $\Lambda$ 7, le Premier Moteur est dit impassible et inaltérable ${ }^{62}$; en $\Lambda 7$ toujours, il n'est pas dit seulement posséder la vie, mais s'identifier à elle ${ }^{63}$, en vertu de son activité autocontemplative. C'est, du reste, cette même activité, qui, en $\Lambda 7$ et 9 , autorise à lui attribuer une existence parfaite ${ }^{64}$ et autarcique. Sans doute est-ce d'ailleurs dans cette autarcie,

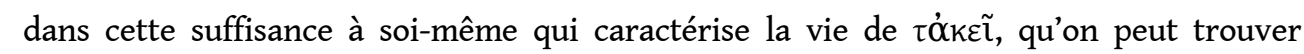


l'élément descriptif qui les rattache le plus fortement à la transcendance, car rien de tel n'est jamais prononcé au sujet des astres, quand c'est, au contraire, l'un des aspects les plus frappants de l'étude des substances immobiles de $\Lambda 7$ et 9. En un sens donc, bien que certains développements du $D C$ puissent s'y prêter, chercher à rattacher aux étoiles fixes ces quelques perfections de 279a20-22 tiendrait presque du forçage, quand la description de l'être ou des êtres transcendants en $\Lambda 7$ et 9 est si parente de notre texte. À tout le moins, on peut conclure que ces quelques éléments d'analyse ne sont pas suffisamment significatifs pour nous forcer à douter de l'évidence textuelle de la transcendance de

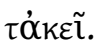

\section{La structure du texte et le problème de son homogénéité}

19 Si le chapitre I 9 se refermait sur les remarques qui précèdent, sans doute n'aurait-il jamais paru énigmatique. Chacun s'accorderait à reconnaître derrière «les êtres de làbas » des réalités hypercosmiques, conformément à ce qu'indiquent clairement à la fois leur description topographique et le contexte argumentatif de l'extrait. Néanmoins, le texte poursuit une analyse dont on a du mal à comprendre comment elle peut ne pas remettre en question l'hypothèse de la transcendance. Il apparaît, en effet, que le texte, à supposer qu'il traite de réalités transcendantes en 279a18-22, change de sujet à une ou plusieurs reprises, pour développer des considérations qui font une référence plus explicite au ciel. À cet égard, trois sous-ensembles textuels peuvent être dégagés.

\section{L'étymologie de l'aíwv (279a22-28)}

Parmi les passages, dont on pourrait estimer qu'ils ruinent l'unité d'un texte consacré à des réalités transcendantes, il y a cette étymologie, par laquelle Aristote justifie qu'on

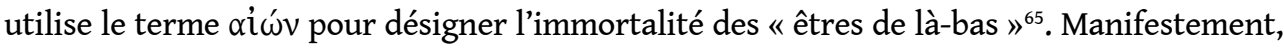
cette remarque porte sur les astres. L'étymologie de l'aíw n'est d'ailleurs pas sans rappeler celle de l'éther en I $3^{66}$. Se pose alors la question de savoir si l'on peut maintenir l'hypothèse de la transcendance de $\tau \dot{\alpha} \kappa \varepsilon \tilde{l}$, dans la mesure où c'est au moyen de la contemplation du ciel et de son immortalité que ce passage entreprend de justifier l'emploi du terme $\alpha i \omega ́ v$ à leur sujet. À ce titre, il semblerait plus naturel que $\tau \dot{\alpha} \kappa \varepsilon \tilde{l}$ désigne les astres fixes: cela conserverait plus d'homogénéité au passage. Aristote expliquerait alors que le terme cíw est parfaitement approprié aux étoiles fixes, dans la mesure où l'immortalité du ciel avait déjà convaincu les Anciens que le vocable qui servirait à signifier la durée de vie de chaque être devrait aussi pouvoir indiquer l'infinité du temps.

Il n'est pas impossible, cependant, qu'on puisse maintenir l'hypothèse de la transcendance, en considérant qu'Aristote, dans ce passage, songe à fonder l'éternité des êtres hypercosmiques sur l'éternité du cosmos lui-même. De fait, si le ciel est immortel, à plus forte raison ce qui le transcende l'est aussi ${ }^{67}$. Surtout, il n'est pas impossible que ce développement constitue une sorte de digression ${ }^{68}$ à mettre entre parenthèses. Cette éventualité découle du fait qu'il ne semble y avoir, dans ce passage sur l'aíw v, aucun référent valable auquel puisse renvoyer ö $\theta \varepsilon v$, en 279a28. À cet égard, son référent le plus évident, et qui maintiendrait le mieux sa signification locale, serait peut-être $\tau \tilde{\omega} v \dot{v} \pi \dot{\varepsilon} \rho$ 


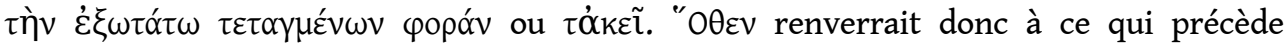
l'analyse étymologique de l'ai $\omega \omega v$, de sorte que celle-ci ne serait, en somme, qu'une digression. C'est là, du moins, l'hypothèse que formule $C$. Natali ${ }^{69}$ qui estime que l'étymologie de l'aíwv, parce qu'elle n'est qu'une parenthèse, n'empêcherait pas ö $\theta \varepsilon v$ de renvoyer à $\tau \dot{\alpha} \kappa \varepsilon \tilde{\text { I }}$ Si tel est le cas, il existe alors peut-être un moyen de comprendre ce passage sans minorer l'évidence manifeste selon laquelle c'est du ciel qu'il tire ses raisons, ni non plus renier la possibilité que « les êtres de là-bas » n'appartiennent pas à l'univers corporel ${ }^{70}$.

\section{Remarques sur la causalité (279a28-30)}

Néanmoins, dire que ö $\theta \varepsilon v$ peut se référer à $\tau \hat{\alpha} \kappa \varepsilon \tilde{l}$, dire que les quelques remarques qui suivent décrivent la fonction principielle de ces réalités, ne présume encore en rien de leur identité. Peut-être se trouve-t-il que la causalité qu'Aristote leur prête (en 279a28-30) n'est intelligible qu'à la condition qu'elle décrive la fonction des astres les plus hauts. En réalité, rien de tel ne ressort de l'analyse de ces trois lignes ${ }^{71}$. Nous pourrions reproduire ici les mêmes remarques que celles que nous avons faites au sujet de la perfection de

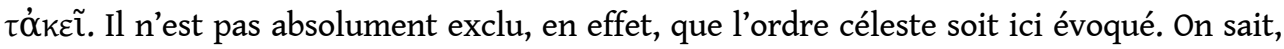
d'une part, que la sphère des fixes est un principe causal éminent ${ }^{72}$. On sait, d'autre part, que certains êtres sont plus proches des astres fixes que certains autres, de sorte que les plus hautes étoiles déterminent l'être et la vie à la fois pour les astres qui leur sont subordonnés et pour les êtres corruptibles. On sait enfin que le ciel ne déploie pas une causalité volontaire - bien qu'elle soit efficiente -, si bien que, en toute rigueur, il est acceptable de dire que la nature lui est suspendue. Il n'en demeure pas moins que la dépendance qui sert à décrire cette causalité, ainsi que le double niveau auquel elle se réalise, ne laissent pas de faire très directement songer au pouvoir causal d'un principe transcendant. En l'occurrence, le verbe $\dot{\varepsilon} \xi \dot{\eta} \rho \tau \eta \tau \alpha l$ rappelle ce passage de Metaph. $\Lambda, 7$ :

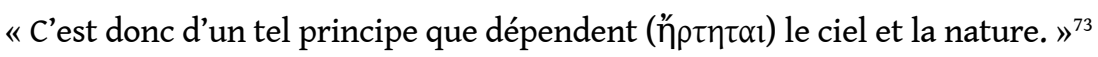

De même, ce double niveau de causalité rappelle ce qu'Aristote montre au sujet de la causalité motrice du Premier Moteur en Phys. VIII, 6 :

À partir de ce qui a été dit, est devenu manifeste ce qui, au début, était pour nous une difficulté, à savoir pourquoi donc tout n'est pas soit en mouvement, soit en repos, ou bien pourquoi certaines choses ne sont pas toujours en mouvement et d'autres toujours en repos, mais pourquoi certaines sont parfois en mouvement et parfois en repos. En effet, la cause de ceci est claire, à présent : c'est parce que les unes sont mues par un être immobile et éternel - c'est pourquoi elles sont toujours en mouvement -, tandis que les autres sont mues par un être mû et changeant, de sorte qu'il est nécessaire qu'elles aussi changent ${ }^{74}$.

Parce que le moteur immobile n'est pas en relation directe avec l'ensemble des corps mus, il sait être une cause d'être et d'éternité pour les uns, de génération et de vie pour les autres. De la sorte, en admettant que ö $\theta \varepsilon v$ se réfère à $\tau \hat{\alpha} \kappa \varepsilon \tilde{\imath}$, il apparaît alors que la causalité que les lignes 279a28-30 décrivent est plus immédiatement intelligible si les « êtres de là-bas » appartiennent à un ordre transcendant de l'existence. Du moins, elles n'apportent aucune raison tangible d'abandonner cette hypothèse. 


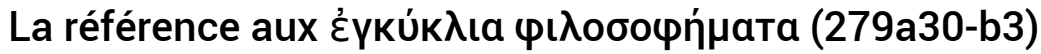
insurmontable, s'il n'y avait, en 279a30-31, cette référence aux « travaux philosophiques destinés au public », dont les interprètes s'accordent en majorité ${ }^{75}$ à penser qu'elle désigne un passage du DP et, plus précisément, ce que restitue Simplicius ${ }^{76}$ dans son commentaire du passage et qu'on désigne à présent comme le fragment 16. C'est sur cet élément que se fonde en partie l'analyse de Jaeger ${ }^{77}$, qui a convaincu que ce texte, dont on doit admettre qu'il est d'une rédaction soignée, est repris du DP. Moraux, qui reprend cette thèse à son compte, estime en conséquence qu'il est impossible que notre texte traite de réalités transcendantes, attendu que cela impliquerait qu'Aristote eût changé trop brutalement de sujet en $279 b 1$, ce qu'interdit la clarté rédactionnelle du passage : «Pour défendre leur exégèse, ces derniers (scil. les partisans de la référence transcendante) sont contraints d'admettre la présence d'une anacoluthe extrêmement dure, et certains d'entre eux vont même jusqu'à modifier le texte traditionnel ; mais le souci d'élégance stylistique qui se manifeste dans tout le passage rend bien improbable l'hypothèse d'une pareille négligence ${ }^{78}$." L'anacoluthe dont parle Moraux est celle qui semble disjoindre les remarques sur l'immutabilité et la perfection du divin, en $279 a$ 30-35, et l'explication, à partir de 279b1, de la continuité éternelle du mouvement astral en raison de la particularité de la translation naturelle de l'éther ${ }^{79}$.

À première vue, il parait évident que les premières lignes du passage servent à justifier, à partir d'une analyse théologique, la perfection que l'analyse cosmologique a permis d'attribuer aux « êtres de là-bas ». La suite du texte, en revanche, traite très clairement du ciel, sans qu'Aristote donne aucun signe qu'il aborde un nouveau sujet. Faut-il donc estimer que, s'il juxtapose ici deux propositions, dont la seconde fait explicitement référence au ciel, c'est que la première le concerne également et, par suite, que $\tau \dot{\alpha} \kappa \varepsilon \tilde{\imath}$ sont les astres fixes?

On sait, toutefois, que certains interprètes ne reculent pas devant cette difficulté et qu'ils admettent parfaitement qu'Aristote ait pu, à partir de 279b1, changer de sujet et passer de la description de la divinité transcendante à celle du ciel. Car c'est un fait que le début du passage (soit 279a30-35) paraît traiter d'une réalité transcendante. N'est-il pas plus logique, en effet, que l'être óplotov, dont Aristote prouve l'existence dans ce fragment 16 et dont il se réclame ici, soit une réalité hypercosmique? N'est-il pas plus évident que celui auquel aucune perfection ne manque soit d'un ordre immatériel et transcendant? Enfin, n'est-il pas légitime de considérer que l'être que rien ne peut mouvoir est une divinité suprême ? C'est pour toutes ces raisons que Simplicius ${ }^{80}$, puis H.-F. Cherniss ${ }^{81}, \mathrm{C}$.

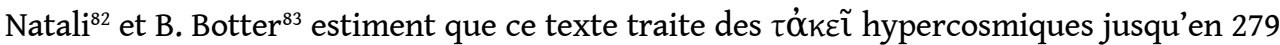
b1, c'est-à-dire jusqu'à ce qu'il soit fait mention d'un mouvement indéfectible du ciel. En somme, 279a18-35 serait, à leurs yeux, une longue parenthèse relative à des êtres transcendants, insérée dans un discours qui commence par traiter du ciel et qui, en 279b1, retourne à son objet premier.

Est-ce à dire, pour autant, qu'il faille, bon an mal an, admettre cette anacoluthe dont Moraux dit qu'il faut la rejeter? Nous ne le croyons pas et Solmsen ${ }^{84}$ a raison, selon nous, de montrer que les remarques sur l'impossibilité que le corps circulaire s'arrête dans un lieu propre n'ont plus aucune justification, si ce qui précède ne s'applique, d'une manière ou d'une autre, à la même réalité. Pourquoi, en effet, Aristote passerait-il ainsi si 
subitement à la question du ciel, sans prendre la peine d'éclairer le sujet de kıveĩ $\alpha \alpha$ par quelques remarques antécédentes? C'est pourquoi Simplicius ${ }^{85}$ plaide en faveur de la

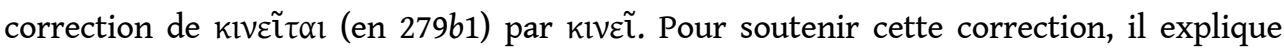
qu'il a déjà rencontré kivẽ̃ dans certains manuscrits. Bien entendu, elle lui permet de mieux défendre la transcendance des "êtres de là-bas» en soutenant qu'ils correspondent, en réalité, à la doctrine du Premier Moteur du ciel. Il y a sans doute peu à dire pour convaincre que cette correction n'est pas incontournable et rares sont les interprètes qui l'acceptent ${ }^{86}$. Disons simplement que non seulement la leçon des manuscrits est la lectio difficilior, mais que, en outre, il n'apparaît pas que la leçon que préconise Simplicius confère une plus grande intelligibilité au texte. Dans l'hypothèse, en

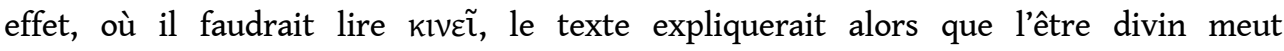
éternellement le ciel, en raison de la nature particulière du déplacement circulaire de l'éther. Rien de cela n'est vraiment cohérent. En tout cas, l'éternité du mouvement céleste, lorsqu'elle est rattachée à l'influence du Premier Moteur, n'a pas besoin de faire mention des aptitudes de l'éther, car ce sont là deux explications différentes. Le ciel est dit se mouvoir éternellement, en vertu soit de l'immutabilité du Premier Moteur, soit de la circularité du déplacement naturel de l'éther ${ }^{87}$. Cette leçon rendrait donc totalement inutile la précision de 27961-3 selon laquelle les astres se meuvent éternellement en raison du mouvement naturel de leur corps élémentaire et non pas en vertu du pouvoir moteur d'une réalité immobile.

Ce refus ne fait qu'accroître la difficulté du passage. Néanmoins, il n'est peut-être pas nécessaire de nier, pour cette raison, que la référence au DP sert en partie à confirmer la perfection des réalités hypercosmiques, ni que c'est de ces réalités qu'il est question à la fin de $D C$ I, 9. Qu'en est-il, en effet, de l'objet immédiat du texte à partir de 279a30 ? C'est du divin que traite ici Aristote, mais d'un divin qui n'est pas autrement déterminé ${ }^{88}$. On pourrait alors envisager qu'Aristote souhaite récapituler au moyen d'une même catégorie de réalité, celle du divin, ce qu'il a dit non seulement à propos des êtres hypercosmiques, dont les lignes 279a30-35 produisent un résumé fort pertinent, mais aussi ce qu'il en est du ciel, au sujet duquel l'étymologie de l'œíwv a montré qu'il était nécessaire qu'il se mût sans pause. Dans cette hypothèse, il n'y aurait à renoncer ni à l'évidence selon laquelle la référence aux « travaux philosophiques destinés au public » sert à prolonger les analyses

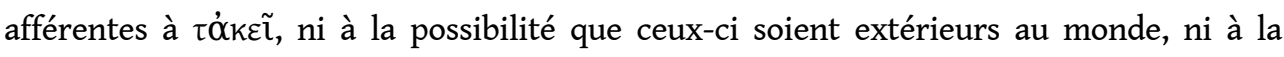
cohérence de l'ensemble de l'extrait. On tiendrait alors un moyen de produire une exégèse uniforme de l'ensemble du passage, en accordant ensemble l'unité argumentative du chapitre, les indices textuels de l'extrait et son homogénéité.

\section{Retour à la question du Premier Moteur Immobile}

Qu'en est-il de la présence de la doctrine du Premier Moteur dans ce texte? Faut-il déduire de ces arguments en faveur de la transcendance cosmique des "êtres de là-bas " que c'est à cette doctrine qu'Aristote fait ici allusion? La question se pose très légitimement car certains de ces arguments ont consisté à faire valoir la parenté qui unit

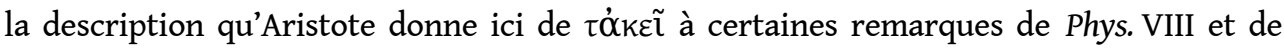
Metaph. $\Lambda$, toutes relatives à la substance immobile. Ce seraient alors l'impassibilité et l'inaltérabilité, la perfection d'une vie éternelle et autarcique, la dépendance de l'ensemble des êtres vis-à-vis d'un principe éminent et même l'incapacité d'être mû qui témoigneraient en faveur de l'identification ou, à tout le moins, en faveur du 


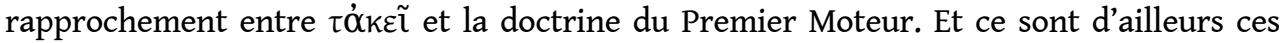
raisons qui motivent la plupart des interprètes à penser ainsi, ceux, du moins, qui penchent du côté de l'hypercosmicité des " êtres de là-bas " ${ }^{89}$. Peut-être à ces arguments une sorte d'habitude s'ajoute-t-elle qui pousse à retrouver le concept de Premier Moteur Immobile partout où l'on peut lire la transcendance divine.

31 À dire vrai, peu d'arguments permettent, à coup sûr, d'estimer que ce texte prolonge les enseignements de Phys. VIII ou de Metaph. $\Lambda$. À cet égard, on pourrait faire valoir que certains éléments semblent interdire qu'on puisse ici retrouver la doctrine du Premier Moteur. C'est le cas, en tout premier lieu, de la multiplicité manifeste des « êtres de làbas ", laquelle multiplicité semble fort peu compatible avec l'unicité du moteur immobile que professe Phys. VIII, $6^{90}$. On pourrait toujours objecter que Metaph. $\Lambda, 8$, affirme l'existence de plusieurs moteurs immobiles, mais il semble fort délicat de supposer que cette doctrine si technique et peut-être tardive trouve un écho dans un texte qu'on estime inspiré par le DP. Peut-être est-ce d'ailleurs dans le DP qu'on peut trouver l'explication de cette multiplicité, dans la mesure où le fragment 17 évoque la possibilité que les principes soient multiples, bien qu'ordonnés ${ }^{91}$. Cependant, cela ne dit rien de précis sur la fonction de ces êtres, rien de plus précis, en tout cas, que le DP lui-même.

Il n'est sans doute pas anodin, non plus, que la fin de $D C$ I, 9 ne prête jamais aucune fonction motrice précise aux « êtres de là-bas ». Il est vrai qu'ils sont ici présentés comme des causes de l'être et de la vie des autres réalités. Cela, toutefois, n'a rien de propre à la doctrine du Premier Moteur. Toute réalité transcendante peut être vaguement qualifiée de principe, comme c'est le cas ici, sans qu'on puisse en déduire qu'il s'agit du Premier Moteur Immobile. Car il y a vraisemblablement une différence non négligeable entre le fait de dire d'un être qu'il est un principe et celui de déterminer avec précision les fonctions principielles qu'il convient de lui attribuer. C'est à cela que se rattache le concept de Premier Moteur Immobile : il ne signifie pas seulement le principe premier ; il dit encore à quel titre il est tel (un moteur premier), sur quels êtres s'exerce son influence (le premier ciel) et quelles voies cette influence emprunte (celle de la causalité finale). Or,

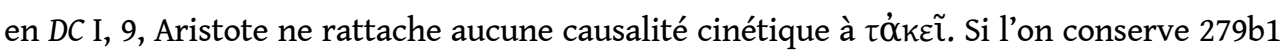
en l'état, alors il n'y a rien de plus à lire que ceci : le ciel doit son mouvement aux

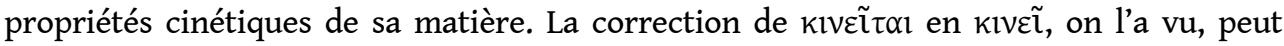
raisonnablement être refusée. De toute façon, elle ne permettrait que très modestement de retranscrire ici la doctrine du Premier Moteur du ciel, dans la mesure où elle n'empêcherait pas de rattacher le mouvement de celui-ci à la nature particulière de l'éther, sans jamais rien dire de la manière particulière qu'a le Premier Moteur de mouvoir. Bien que Cherniss ${ }^{92}$ estime qu'on retrouve, dans ce texte du $D C$, la doctrine de la causalité finale, il semble hasardeux de le suivre sur ce point. Son interprétation se fonde sur la conviction que le DP développait déjà la thèse d'une cause finale motrice du ciel. Il

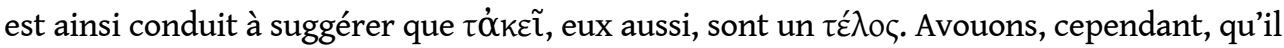

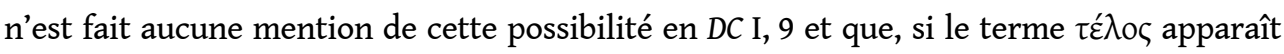
bel et bien en $279 a 23$ et 27 , ce n'est pas dans le but de désigner une finalité ${ }^{93}$. Même la situation hypercosmique de $\tau \hat{\alpha} \kappa \varepsilon \tilde{\imath}$ ne semble pas faire référence à une quelconque fonction cinétique, quand on se souvient que c'est vraisemblablement en vue d'expliquer la plus grande rapidité de la translation du premier ciel qu'Aristote situe le moteur immobile sur la circonférence du cercle ${ }^{94}$, manifestant en cela une plus grande précision et une plus grande attention à l'influence motrice de l'être transcendant ${ }^{95}$. 
pourrait toujours objecter à cette analyse que le silence d'Aristote, sur la fonction motrice des réalités de là-bas, ne prouve rien, étant donné que la description qu'il donne de ces êtres est trop proche de celle qu'il développe au sujet du Premier Moteur. Néanmoins, force est de constater que cela n'a rien de spécifique à cette doctrine de dire qu'un être incorporel et transcendant jouit ipso facto d'une parfaite impassibilité, qu'il est inaltérable et éternel et qu'il mène pour cette raison une existence qu'aucun manque ne vient troubler. En tout état de cause, c'est la logique de l'incorporéité qui justifie les remarques de 279a18-22 et c'est celle, complémentaire, de la perfection divine qui motive les développements de 279a30-35. Rien de tout cela ne provient d'une analyse minutieuse des qualités qui doivent nécessairement être celles d'un moteur premier et immobile. Assurément, les «êtres de là-bas » sont divins et peut-être hypercosmiques : c'est pour cette raison que leurs qualités ressemblent fort à celles du Premier Moteur. Cela ne signifie pas pour autant qu'on les puisse identifier car la spécificité du concept de Premier Moteur ne se résume pas aux propriétés divines, et assez convenues, qui sont les siennes, mais à l'originalité de la dynamique argumentative qui justifie qu'on les lui accorde ${ }^{96}$. En l'occurrence, cette dynamique argumentative, celle de la fonction principielle du moteur premier et immobile, n'est jamais mobilisée dans ce texte de $D C$ I, 9 .

iissons que nous ne savons presque rien de ces «êtres de là-bas " ${ }^{97}$, si ce n'est que plusieurs indices convergent pour montrer qu'ils sont vraisemblablement transcendants à la sphère des fixes et qu'aucune fonction précise ne leur est clairement assignée. Sans doute sont-ils des réalités divines admirables, des principes premiers et universels, mais il demeure que nous ne savons ni à quel titre ils le sont, ni comment se rattachent à eux les autres êtres. Dans ces conditions, on peut estimer qu'il n'est pas définitivement certain qu'on puisse lire ce texte comme une allusion rapide et implicite qu'Aristote ferait à sa doctrine du Premier Moteur Immobile. C'est pourquoi nous pouvons douter, conformément à ce que nous suggérions plus haut, que la signification première de celleci soit d'ordre théologique, avant que d'être archéologique. Parce que ce texte de $D C$ I, 9 témoigne de l'existence de divinités hypercosmiques, mais peut-être non motrices, la singularité même de la doctrine du Premier Moteur ne consiste vraisemblablement pas à proposer l'existence d'une réalité divine, parfaite et autarcique, ni celle d'un principe premier et transcendant, mais, plus spécifiquement, à éclairer la fonction causale réelle de ce principe, en fournissant une explication détaillée de la relation qui l'unit au ciel et à la nature.

\section{BIBLIOGRAPHIE}

ALLAN, D.J., 1936 : Aristotelis De caelo libri quattuor, recognovit brevique adnotatione critica instruxit ALLAN, D.J., Oxford, 1936 (Scriptorum classicorum bibliotheca oxoniensis).

BERTI, E. 1977 : Aristotele : dalla dialettica alla filosofia prima, Padoue, 1977 (Saggi di una nuova storia della filosofia). [Rééd. Milan, 2004 (Il pensiero occidentale).] 
BODEÜs, R. 1992 : Aristote et la théologie des vivants immortels, Montréal-Paris, 1992 (NoêsisCollection d'études anciennes).

BOTTER, B. 2005: Dio e divino in Aristotele, Sankt Augustin, 2005 (International Aristotle Studies, 3). CHERNISS, H.-F. 1944, Aristotle's Criticism of Plato and the Academy, 1, Baltimore (MD), 1944. Dumoulin, B. 1981 : Recherches sur le premier Aristote : Eudème, De la philosophie, Protreptique, Paris, 1981 (Bibliothèque d'histoire de la philosophie).

ELDERS, L.J. 1966: Aristotle's Cosmology: a Commentary on the De Cœlo, Assen, 1966 (Institute for Ancient and Mediaeval Philosophy. Philosophical Institute at the University of Utrecht. Philosophical texts and studies, 13).

HEIBERG, J. L. 1894 : Simplicii In Aristotelis De caelo commentaria, edidit HEIBERG, J. L., Berlin, 1894 (Commentaria in Aristotelem Graeca, 7).

GUTHRIE, W.K.C. 1933: « The Development of Aristotle's Theology. 1 », The Classical Quarterly, 27 (1933), p. 162-171.

GUTHRIE, W.K.C. 1939: Aristotle in Twenty-three volumes. 6, On the Heavens, with an English Translation, Cambridge (Mass.)-Londres, 1939 (The Loeb Classical Library, 338).

JAEGER, W. 1957: Aristotelis Metaphysica, recognovit brevique adnotatione critica instruxit JAEGER, W., Oxford, 1957 (Scriptorum classicorum bibliotheca Oxoniensis).

JAEGER, W. 1997 : Aristote : fondements pour une histoire de son évolution, Paris, 1997 (Polemos, 13). [Traduction, par O. Sedeyn, de Aristoteles : Grundlegung einer Geschichte seiner Entwicklung, Berlin, 1923.]

LONGO, O. 1962 : Aristotele, De caelo, Introduzione, testo critico, traduzione e note di -, Florence, 1962 (Classici greci e latini con testo a fronte. Seconda serie. Opere di Aristotele, 1).

MERLAN, Ph. 1966 : « Two Theological Problems in Aristotle's Met. Lambda 6-9 and De cœlo A, 9 », Apeiron, 1 (1966), p. 3-13.

MORAUX, P. 1965 : Aristote, Du ciel, Paris, 1965 (Collection des Universités de France).

MOREAU, J. 1939 : L'Âme du monde de Platon aux stoïciens, Paris, 1939 (Collection d'études anciennes). MUGNIER, R., 1930 : La Théorie du premier moteur et l'évolution de la pensée aristotélicienne, Paris, 1930 (Bibliothèque d'histoire de la philosophie).

NATALI, C. 1974 : Cosmo e divinità : la struttura logica della teologia aristotelica, L'Aquila, 1974 (Methodos, 6).

PELLEGRIN, P. \& C. DALIMIER 2004 : Aristote, Traité du ciel, traduction et notes par PELLEGRIN, P. \& C. DALIMIER, introduction par P. P., Paris, 2004 (GF 1036).

PEPIN, J. 1964 : Théologie cosmique et théologie chrétienne (Ambroise, Exam. I, 1, 1-4), Paris, 1964

(Bibliothèque de philosophie contemporaine. Histoire de la philosophie et philosophie générale). Ross, W.D. 1936: Aristotle's Physics, A Revised Text with Introduction and Commentary, Oxford, 1936.

Ross, W.D. 1950: Aristotelis Physica, recognovit brevique adnotatione critica instruxit Ross, W.D., Oxford, 1950 (Scriptorum classicorum bibliotheca Oxoniensis). Aristote, De Cœlo, I 9203

ROSS, W.D. 1955: Aristotelis Fragmenta selecta, recognovit brevique adnotatione instruxit Ross, W.D., Oxford, 1955 (Scriptorum classicorum bibliotheca oxoniensis). 
SOLMSEN, F. 1960: Aristotle's System of the Physical World: a Comparison with his Predecessors, Ithaca, N.Y., 1960 (Cornell Studies in Classical Philology, 33).

SOLMSEN, F. 1976: « Beyond the Heavens », Museum Helveticum, 33 (1976), p. 24-32.

SтоскS, J.L. 1930: De cœlo, dans: W.D. Ross (éd.), The Works of Aristotle Translated into English. 2, Oxford, 1930.

TRICOT, J. 1949 : Aristote, Traité du ciel, traduction et notes, Paris, 1949 (Bibliothèque des textes philosophiques).

VITELLI, G. 1897 : Ioannis Philoponi in libros De generatione et corruptione commentaria, edidit VITELLI, G., Berlin, 1897 (Commentaria in Aristotelem Graeca, 14.2).

WERNER, Ch., 1910 : Aristote et l'idéalisme platonicien, Paris, 1910 (Collection historique des grands philosophes).

ZELLER, E. 1862 : Die Philosophie der Griechen in ihrer geschichtlichen Entwicklung. II. 2, Aristoteles und die alten Peripatetiker, Tübingen, 1862.

\section{NOTES}

1. De Colo (DC), I, 9, 278a23-b9.

2. Tel est l'objectif explicite de I 8-9. Cf. $276 a 18$ sqq. et $277 b 26$ sqq.

3. $D C$ I, 9, 278b25-279a5.

4. DC 279a11-18.

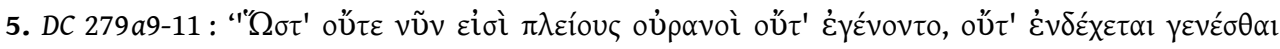

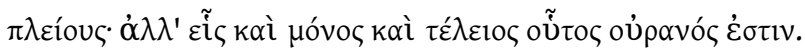

6. $D C \mathrm{I}, 3,27064-25$.

7. $D C$ I, 9, 279a18-b3.

8. $D C 279 a 22$ sqq.

9. $D C 279 a 30 s q q$.

10. En faveur de la première hypothèse, penchent ensemble Alexandre d'Aphrodise (apud Simplicius, in De cœlo, 287, 21 sqq. Heiberg 1894), Werner 1910, p. 328 sqq., Mugnier 1930, p. 77-78, Moreau 1939, p. 119, ou Moraux 1965, p. LXXV.

11. C'est cette option que défendent les études de Simplicius (in De cœlo, 290, 4 sqq. Heiberg), Zeller 1862, p. 364, n. 6., Jaeger 1997, p. 311, Ross 1936, p. 97, Guthrie 1933, p. 168, Solmsen 1960, p. 308, n. 20, Cherniss 1944, p. 587-588, Merlan 1966, p. 9-13, Berti 1977, p. 439, Natali 1974, p. 146-151 et Dumoulin 1981, p. 53-63.

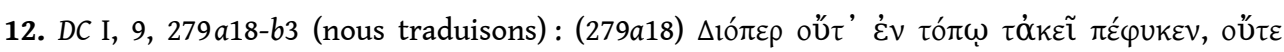

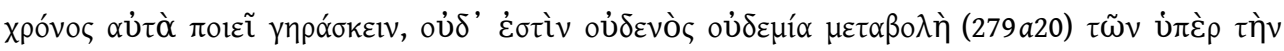

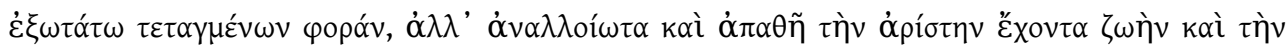

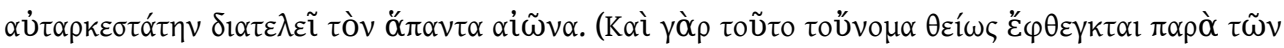

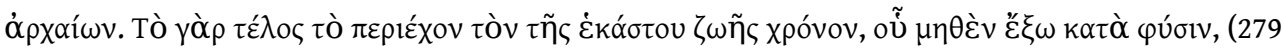

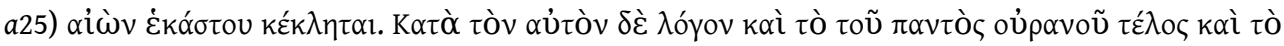

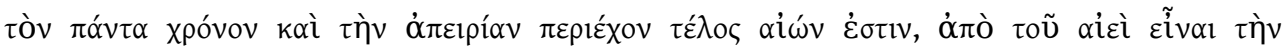

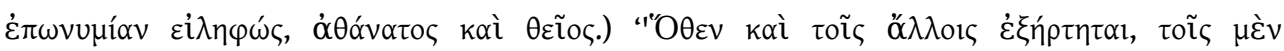

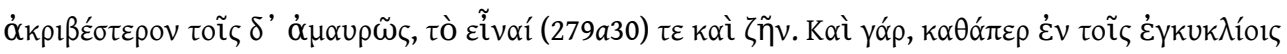

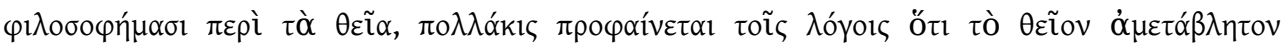

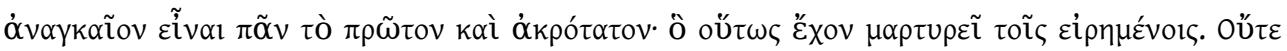

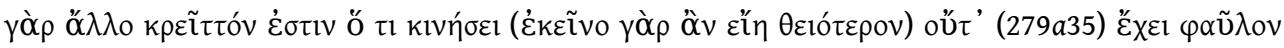




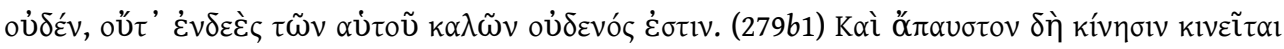

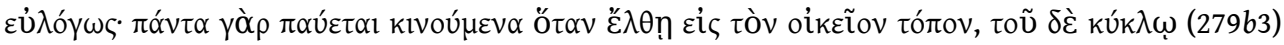

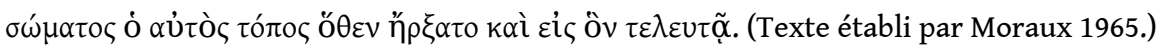

13. On s'est beaucoup interrogé sur l'unité thématique du traité : Alexandre d'Aphrodise estime qu'il traite du ciel, dans les trois sens qu'Aristote confère au terme oủpavóc, à savoir la sphère des fixes, le ciel tout entier et le monde (apud Simplicius, in De cœlo, 1, 2-24 H.). D'une façon sensiblement plus restrictive, Syrianus considère qu'il est tout entier consacré à l'étude de l'éther (ap. Simplicius, in De colo, 1, 24-2, 16 H.). Simplicius, quant à lui, y voit une étude exhaustive des cinq corps élémentaires (in De cœlo, 4, 25-5, $24 \mathrm{H}$.), tandis que Philopon estime qu'il procède à l'étude des corps naturels éternels (In libros de generatione et corruptione commentaria, 1, 13-23 Vitelli). Sans doute le plus prudent et le plus exact est-il de suivre Pellegrin \& Dalimier 2004, p.31-38, qui montrent que tous les développements du traité contribuent à une étude proprement cosmologique.

14. Plusieurs interprètes, cependant, en l'occurrence Moraux 1965 p. XLIII-XLIV, et Pellegrin \& Dalimier 2004, p. 45, estiment que le $D C$, dans la mesure où il est un traité de cosmologie, n'a pas à faire référence aux réalités transcendantes ni au moteur immobile, de sorte que son silence sur la question du Premier Moteur ne serait pas le signe d'une variation doctrinale, mais d'une régionalisation stricte du propos.

15. DC II, 6, 288a27-b7.

16. DC II, 12, 292a18-b25.

17. C'est ainsi, à tout le moins, que les considère Guthrie 1939, p. XXIV-XXV, dans son introduction au traité.

18. À la première éventualité se rattache, par exemple, l'exégèse du passage que produit Moreau 1939, p. 119 ; à la seconde, celle, plus communément partagée, de Jaeger 1997, p. 311, de Cherniss 1944, p. 587-588 ou de Berti 1977, p. 439.

19. Exceptions notables, cependant, qui sont celles des interprétations de Merlan 1966, p. 9-13, ou de Solmsen 1960, p. 308, n. 20.

20. Du reste, ce problème complète la preuve de la finitude des corps élémentaires et de l'univers, en DC I, 5-7, car, pour prouver que le monde est fini, il faut, d'une manière ou d'une autre, démontrer qu'il est unique.

21. Dans le cas, en effet, où plusieurs mondes existeraient, il faudrait cependant admettre qu'ils sont constitués des mêmes corps simples que le nôtre. Ces corps simples seraient donc animés des mêmes mouvements naturels et leurs lieux propres seraient, par suite, identiques à ceux des éléments de notre monde. De la sorte, la terre de l'autre monde se porterait vers le centre du nôtre, si bien que, au regard du monde extérieur, elle serait portée vers le haut. La théorie des lieux naturels interdit pareille absurdité (276a22-b21). Aussi faut-il estimer que, même dans l'hypothèse de mondes multiples, les lieux naturels des corps élémentaires de même nature sont numériquement uniques. Si tel est le cas, si la terre de notre monde et celle de tous les autres n'ont qu'un seul lieu propre, c'est donc qu'il n'existe qu'un seul centre, qu'un seul monde (276b 26-277a12).

22. DC I, 9, 278a18-21.

23. $D C$ I, 9, 278a23-28.

24. Ce ressort démonstratif en faveur de l'unicité de l'univers exploite manifestement l'argumentation platonicienne du Timée : de même que Platon, en 32c-33b, explique que le monde est formé de la totalité des quatre éléments, de même Aristote s'appuie ici sur l'idée que l'univers est constitué de la totalité de la matière existante, de telle sorte qu'il ne peut rester aucun corps capable d'entrer dans la composition d'un second univers.

25. C'est un argument que le chapitre 8 a déjà timidement exploité, en montrant, d'une part, que la théorie des lieux naturels implique que les mouvements naturels aient un terme et qu'ils ne puissent se prolonger à l'infini en dehors de l'univers (277a20-b9), en expliquant, d'autre part, 
que ni le corps dont le lieu propre est l'extrémité du ciel (l'éther), ni celui qui se trouve dans le lieu intermédiaire (l'air ou le feu) ne peuvent résider en dehors de l'univers. Pour le premier, en effet, il est contradictoire de penser que le corps qui délimite l'univers puisse être à l'extérieur de cette limite, pour le second, attendu qu'il possède une pesanteur, il est impossible qu'il s'élève au-dessus de l'éther qui n'est ni lourd ni léger (277b12-24).

26. Et jusqu'en $279 a 5$.

27. La preuve en a été faite en $D C$ I, 3, 270b26-31.

28. Celle de l'incorruptibilité du monde.

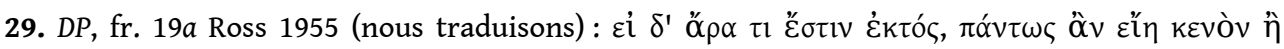

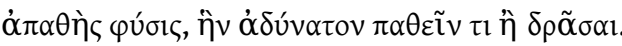

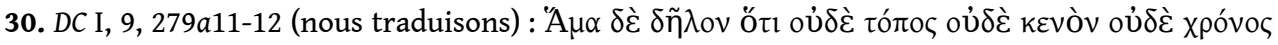

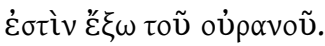

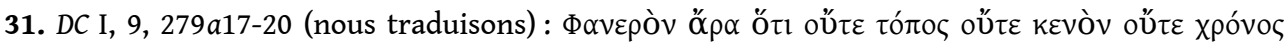

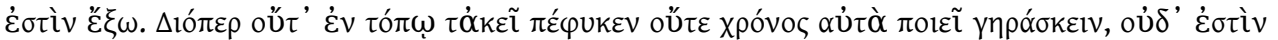

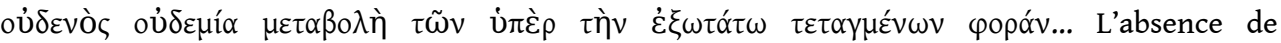
changement est liée à celle du temps (et inversement), si bien qu'on peut inclure dans un même examen le fait que les « êtres de là-bas » ne sont pas dans un lieu, qu'ils ne vieillissent pas dans le temps et qu'ils ne connaissent aucun changement. Chacune de ces caractéristiques est une conséquence de l'absence de corps en dehors de l'univers.

32. Jaeger 1997, p. 309.

33. Guthrie 1939, p. 90.

34. Allan 1936.

35. Simplicius, in De colo, 290, 1-17 H. Natali 1974, p. 146, met en valeur le même argument: la

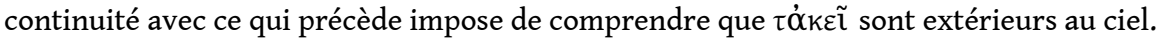

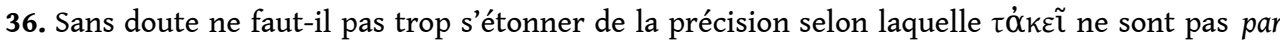
nature ( $\pi \varepsilon \dot{\varphi} \varphi \kappa \varepsilon v$ ) dans un lieu : cette remarque n'implique peut-être pas que les êtres dont il s'agit sont naturels, mais qu'il n'est pas dans leur nature d'admettre les déterminations de lieu et de temps, lesquelles caractérisent n'importe quel corps.

37. C'est en ce sens que Natali 1974, p. 145, remarque que ces quelques mots traduisent une manière physique de dire la transcendance.

38. Phys. IV, 14, $223 \mathrm{~b} 12$ sqq.

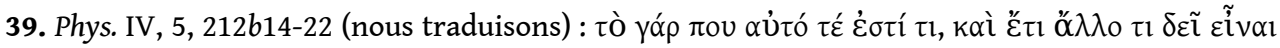

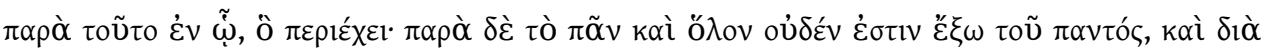

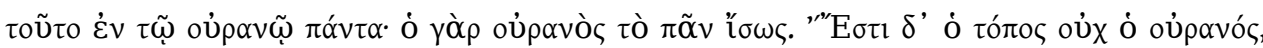

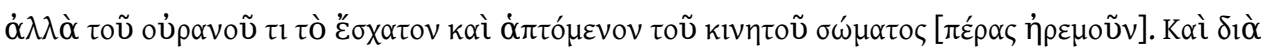

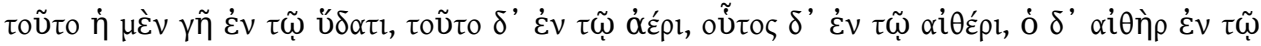

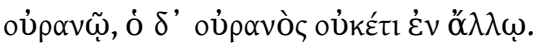

40. DC I, 3, 270a13-22.

41. DC II, 1, 284a13.

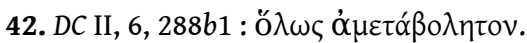

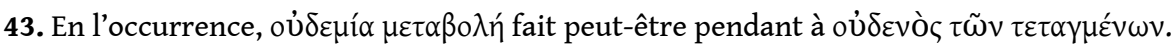

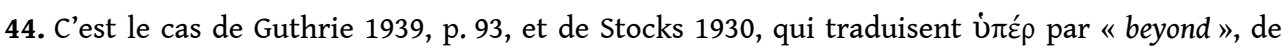
Tricot 1949, p. 45, et de Pellegrin \& Dalimier 2004, p. 147, qui traduisent par «au-dessus », de Longo 1962 qui donne « al di là della orbita più esterna » ou de Botter 2005, p. 149, qui choisit «al di là della traslazione più esterna ".

45. Moraux 1965, p. 37 : «il n'est point de changement pour aucun des êtres disposés sur la translation la plus extérieure... » (nous soulignons).

46. Apud Simplicius, in De colo, 288, 1-8 H.

47. Comme c'est le cas en $278 b 25$ ou en $279 a 18$. 
48. Citons, par exemple, $D C 278 b 13,278 b 15,289 a 28-29,289 b 17,292 a 11,293 a 6-7$.

49. Par exemple $278 b 12-13$; 278b16-17 ; 278b20 ; 278b21-22 ; 278b25 ; $291 a 35$.

50. Entre autres exemples : $276 b 13 ; 276 b 20 ; 276 b 32 ; 277 a 10 ; 277 b 16 ; 278 b 14$.

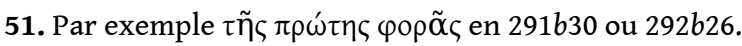

52. Apud Simplicius, in De cœlo, 288, 1-8 H. 53.

53. Cf. $291 b 30$ ou $292 b 26$.

54. Solmsen 1976, p. 29-30.

55. $D C$ I, 3, 270a14 ; 270a25-35; 270b2.

56. DC I, 3, 270b2 ; II, 1, 284a14.

57. DC II, 1, 284a15 : ö́rovo५.

58. DC II, 1, 284a17-18.

59. DC II, 12, 292a20-21.

60. DC II, $1,284 a 29$.

61. DC II, 1, 289a32.

62. Metaph. 1073a11.

63. Metaph. $1072 b 14 ; 1072 b 26-30$.

64. Metaph. $1072 a 19 ; 1072 a 35 ; 1072 b 14-15 ; 1072 b 24 ; 1072 b 28-30 ; 1072 b 32 ; 1074 b 20 ; 1074 b 33$.

65. Voir le texte et la traduction du passage, p. 178.

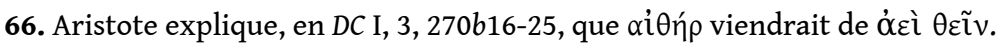

67. Ce serait alors à une sorte d'argument a fortiori que l'on assisterait, fort semblable à l'argumentation que Botter 2005, p. 30 sqq., dit caractériser les réflexions aristotéliciennes sur le divin.

68. C'est pourquoi nous le donnons entre parenthèses, suivant, en cela, l'exemple de Moraux 1965, de Natali 1974, p. 148, et de Botter 2005, p. 150-151.

69. Natali 1974, p. 149.

70. Solmsen 1960, p. 308, n. 20, de son côté, suggère que le texte, à partir de 279a25 (soit à partir

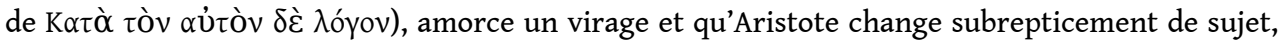
sans que cela ruine pour autant la réalité de la transcendance de $\tau \dot{\alpha} \kappa \varepsilon \tilde{l}$.

71. Voir le texte et la traduction du passage, supra $p .178$.

72. DC II, 12, 292b28-30.

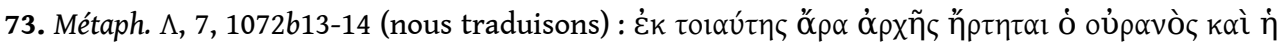

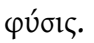

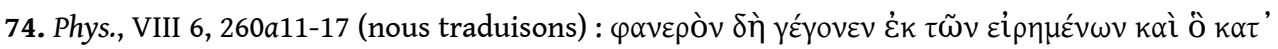

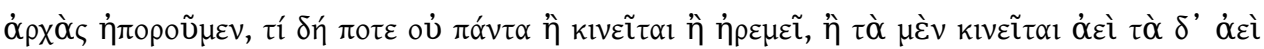

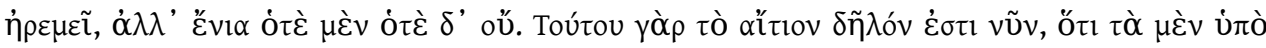

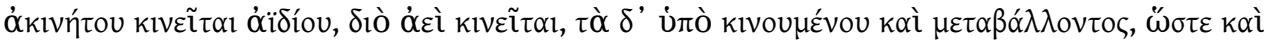

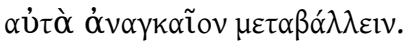

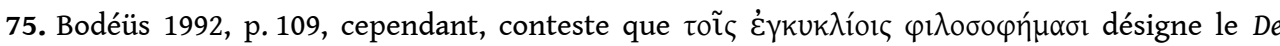
Philosophia. Aristote, estime-t-il, ne peut attendre une confirmation de ses propres écrits. C'est pourquoi la référence viserait, en réalité, le Timée. Merlan 1966, p.13, avait déjà formulé ce problème, sans indiquer d'autres référents possibles. En raison du même argument, mais aussi en raison de la pluralité des travaux mentionnés, Botter 2005, p. 152, estime qu'Aristote fait plus largement référence à une tradition présocratique. Notons cependant que le commentaire de Simplicius (in De coelo, 288, 28-289, 2 H.) montre très clairement que ce passage est une reprise, parfois littérale, du fragment 16 . Notons, en outre, que le DP apporte vraiment une confirmation aux thèses développées au début de l'extrait, dans la mesure où il confirme, d'un point de vue théologique, ce qu'une réflexion cosmologique a permis de mettre en évidence. Un type de discours témoigne ainsi en faveur d'un autre. Du reste, la pluralité des travaux mentionnés peut encore s'expliquer par le consensus qu'Aristote est en droit de trouver entre ses propres 
démonstrations du DP et celles d'autres auteurs, comme Platon, par exemple, auquel Simplicius renvoie à la fin du fragment 16, pour indiquer qu'Aristote s'est fortement inspiré de République, II.

76. Simplicius, in De colo, 288, 28 sqq. H.

77. Jaeger 1997, p. 311-312. Jaeger estime même que c'est la totalité de l'extrait (soit 279a18-b3) qui est issue du DP. Cette hypothèse, cependant, met à mal la continuité indiscutable des premières lignes du passage avec les remarques de 279a11-18. Il semble donc que le plus raisonnable soit qu'Aristote ne s'inspire de son dialogue qu'à partir de $279 a 30$.

78. Moraux 1965, p. LXXV.

79. Voir le texte et la traduction du passage, supra p. 178-179.

80. Simplicius, in De cœlo, 291, 10-24 H.

81. Cherniss 1944, p. 587-588.

82. Natali 1974, p. 150.

83. Botter 2005, p. 153.

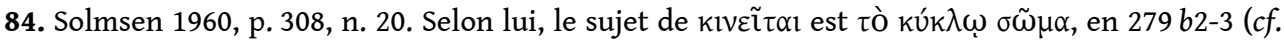
1976, p. 29, n. 16).

85. Simplicius, in De cœlo, 291, 25-29 H.

86. C'est le cas de Tricot 1949, p. 45, n. 3, et p. 46, n. 4.

87. C'est du moins ce qu'enseigne la double démonstration de l'éternité du mouvement astral en Phys. VIII : Aristote y établit qu'il existe un mouvement continu et éternel, en recourant soit à l'immobilité et à l'immutabilité du Premier Moteur, en VIII, 5-6, soit à l'analyse de la translation circulaire en VIII, 8-9.

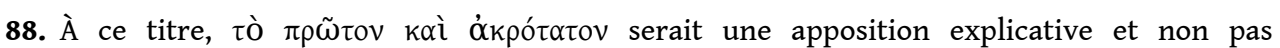
déterminative.

89. C'est le cas de Simplicius (in De cœlo, 290, 1-20 H.), de Zeller 1862, p. 364, n. 6, de Jaeger 1997, p. 311, de Ross 1936, p. 97, de Cherniss 1944, p. 587-588, de Berti 1977, p. 439, de Natali 1974, p. 148 sqq. et de Dumoulin 1981, p. 55 ou de Botter 2005, p. 150.

90. Phys. VIII, 6, 259a6-20.

91. C'est l'hypothèse interprétative que formule Dumoulin 1981, p. 54.

92. Cherniss 1944, p. 588.

93. La question de la présence de la doctrine du Premier Moteur dans le DP est d'ailleurs loin d'être établie.

94. Phys. VIII, 10, 267b6-9.

95. C'est pour toutes ces raisons que Merlan 1966, p. 13, ou Solmsen 1960, p. 308, n. 20, et 1976, p. 29, qui pourtant s'accordent sur la transcendance de $\tau \dot{\alpha} \kappa \varepsilon \tilde{l}$, refusent de voir en eux une quelconque allusion à la doctrine du Premier Moteur. Elders 1966, p. 144, va plus loin, dans ce sens, en faisant l'hypothèse que ces êtres hypercosmiques et non moteurs seraient analogues aux Idées platoniciennes avec lesquelles Aristote n'aurait pas encore rompu. Pour défendre cette hypothèse, il explique que l'argumentation précédente, à l'occasion de laquelle Aristote fait une distinction entre la forme elle-même et la forme incarnée dans la matière, a pu l'amener à traiter ensuite de la question des formes séparées. En elle-même, la continuité argumentative que souhaite faire valoir Elders est assez pertinente. Il n'est certes pas impossible que le contexte de ce passage, à savoir la démonstration de l'unicité du ciel, ait pu convaincre Aristote de se pencher sur le problème des formes immatérielles. En revanche, tout porte à croire, comme le montre très bien Guthrie 1933, p. 168, que, si tel est vraiment le cas, si c'est bien aux formes séparées qu'Aristote fait ici allusion, ce ne peut être alors que dans une visée critique qu'il y prête attention, son opposition à la théorie des Idées étant déjà parfaitement évidente dans le DP. Rien, toutefois, ne permet de détecter dans ce texte une quelconque rhétorique polémique, puisque les « êtres de là-bas » sont dits être des réalités éternelles et bienheureuses, ce qui, s'il s'agissait des Idées, paraîtrait tout à fait incongru. 
96. En Phys. VIII, par exemple, c'est l'existence d'un mouvement éternel et continu qui encourage à énoncer l'immobilité du Premier Moteur (VIII, 5), son éternité, son unicité et son immutabilité (VIII, 6), ainsi que le fait qu'il ne comporte ni grandeur ni parties (VIII, 10).

97. Nous ne dirons rien de l'hypothèse de Pépin 1964, p. 161-170, qui soutient que la divinité en question est l'éther hypercosmique. Peu d'éléments, en effet, sont en faveur de cette thèse et ce tout particulièrement dans le $D C$ : J. Pépin lui-même ne reconnait-il pas qu'il n'est qu'un seul texte, dans ce traité, en faveur de son hypothèse?

\section{RÉSUMÉS}

Il est relativement peu de textes qui, dans le De Colo, témoignent de l'existence de réalités incorporelles transcendantes à l'ordre astral. La conclusion, sur laquelle se referme la démonstration de l'unicité du ciel en I 9, est-elle de ceux-là ? Les « êtres de là-bas » y désignentils les réalités sidérales les plus hautes ou certaines instances hypercosmiques? C'est à l'identification de ces êtres qu'il s'agit ici de procéder. En montrant que convergent ensemble les indices textuels du passage et son homogénéité démonstrative, il pourra alors apparaître que ces êtres, dont Aristote vante l'immutabilité et la perfection, transcendent l'ordre céleste, sans qu'on puisse toutefois déterminer la relation exacte qui les unit au ciel, obérant ainsi la possibilité de lire, à travers eux, une référence implicite à la doctrine du Premier Moteur Immobile.

In the De Colo, there are few texts in which Aristotle gives evidence for the existence of some incorporeal and transcendental beings. Is the conclusion of the proof of the uniqueness of the world, in I9, one of these texts? Do $\tau \dot{\alpha} \kappa \varepsilon \tilde{~ i n d i c a t e ~ t h e r e ~ t h e ~ h i g h e s t ~ s t a r s ~ o r ~ c e r t a i n ~}$ supramondane realities? This study tries to determine the identity of these $\tau \dot{\alpha} \kappa \varepsilon \tilde{l}$, by showing that both textual analysis of the passage and investigation of his structure may convince that these beings, which are said immutable and perfect, are located beyond the highest heavenly sphere. Nevertheless, it does not appear that Aristotle ever clearly define their connection to the heaven, which suggests that it is impossible to associate them to the Prime Mover.

\section{INDEX}

Mots-clés : astronomie, métaphysique, Premier Moteur

Keywords : astronomy, metaphysics, Prime Mover

\section{AUTEURS}

\section{FABIENNE BAGHDASSARIAN}

Université Lyon 3 